\title{
Pretreatment growth rate as a predictor of tumor control following Gamma Knife radiosurgery for sporadic vestibular schwannoma
}

\author{
Alexander P. Marston, MD, ${ }^{1}$ Jeffrey T. Jacob, MD, ${ }^{2}$ Matthew L. Carlson, MD, ${ }^{1}$ \\ Bruce E. Pollock, MD, ${ }^{2,3}$ Colin L. W. Driscoll, MD,1,2 and Michael J. Link, MD ${ }^{1,2}$ \\ Departments of ${ }^{1}$ Otolaryngology-Head and Neck Surgery, ${ }^{2}$ Neurologic Surgery, and ${ }^{3}$ Radiation Oncology, Mayo Clinic School of \\ Medicine, Rochester, Minnesota
}

\begin{abstract}
OBJECTIVE Over the last 30 years, stereotactic radiosurgery (SRS) has become an established noninvasive treatment alternative for small- to medium-sized vestibular schwannoma (VS). This study aims to further define long-term SRS tumor control in patients with documented pretreatment tumor growth for whom conservative observation failed.
\end{abstract}

METHODS A prospective clinical database was queried, and patients with sporadic VS who elected initial observation and subsequently underwent SRS after documented tumor growth between 2004 and 2014 were identified. Posttreatment tumor growth or shrinkage was determined by $\mathrm{a} \geq 2-\mathrm{mm}$ increase or decrease in maximum linear dimension, respectively.

RESULTS Sixty-eight patients met study inclusion criteria. The median pre- and posttreatment observation periods were 16 and 43.5 months, respectively. The median dose to the tumor margin was $13 \mathrm{~Gy}$ (range 12-14 Gy), and the median maximum dose was 26 Gy (range 24-28 Gy). At the time of treatment, 59 tumors exhibited extracanalicular (EC) extension, and 9 were intracanalicular (IC). Of the 59 EC VSs, 50 (85\%) remained stable or decreased in size following treatment, and $9(15 \%)$ enlarged by $>2 \mathrm{~mm}$. Among EC tumors, the median pretreatment tumor growth rate was 2.08 $\mathrm{mm} / \mathrm{year}$ for tumors that decreased or were stable, compared with $3.26 \mathrm{~mm} / \mathrm{year}$ for tumors that grew following SRS ( $p$ $=0.009$ ). Patients who demonstrated a pretreatment growth rate of $<2.5 \mathrm{~mm} / \mathrm{year}$ exhibited a $97 \%$ tumor control rate, compared with $69 \%$ for those demonstrating $\geq 2.5 \mathrm{~mm} / \mathrm{year}$ of growth prior to SRS ( $p=0.007$ ). No other analyzed variables were found to predict tumor growth following SRS.

CONCLUSIONS Overall, SRS administered using a marginal dose between 12-14 Gy is highly effective in treating VSs in which initial observation fails. Tumor control is achieved in $97 \%$ of VSs that exhibit slow $(<2.5 \mathrm{~mm} /$ year $)$ pretreatment growth; however, SRS is less successful in treating tumors exhibiting rapid growth ( $\geq 2.5 \mathrm{~mm} / \mathrm{year})$.

https://thejns.org/doi/abs/10.3171/2016.5.JNS153013

KEY WORDS acoustic neuroma; vestibular schwannoma; Gamma Knife radiosurgery; stereotactic radiosurgery

$\mathrm{T}$ He optimal treatment of sporadic, small- to medium-sized vestibular schwannoma (VS) remains highly controversial. ${ }^{4,8}$ Some centers use up-front proactive therapy, believing that this strategy provides the best chance of long-term tumor control and preservation of function. ${ }^{15,22}$ Others advocate for an initial so-called wait-and-scan approach, understanding that many VSs do not grow for extended periods of time. ${ }^{24}$ The goal of mi- crosurgery is complete tumor removal and cranial nerve preservation. In contrast, successful tumor control with stereotactic radiosurgery (SRS) is variably defined as lack of growth after treatment, lack of symptomatic growth, freedom from additional treatment, and (more narrowly) as freedom from additional nonradiation treatment, allowing for repeat radiation if necessary. Because the natural history of VS predicts that many untreated tumors will

ABBREVIATIONS AAO-HNS = American Academy of Otolaryngology-Head and Neck Surgery; EC $=$ extracanalicular; GKS = Gamma Knife radiosurgery; IC $=$ intracanalicular; IQR = interquartile range; $S R S$ = stereotactic radiosurgery; VS = vestibular schwannoma.

ACCOMPANYING EDITORIAL See pp 374-379. DOI: 10.3171/2016.7.JNS161236.

SUBMITTED December 24, 2015. ACCEPTED May 13, 2016.

INCLUDE WHEN CITING Published online November 25, 2016; DOI: 10.3171/2016.5.JNS153013. 
remain quiescent during many years of follow-up, it has been suggested that the true efficacy of SRS can only be accurately appraised after analyzing treatment outcomes of tumors that have demonstrated definite radiological growth prior to SRS. ${ }^{12,16}$

Stereotactic radiation therapy for VS can be delivered in a single fraction, as SRS; as hypofractionated stereotactic radiation therapy in 2-5 sessions; or with more conventional fractionation schemes, usually over a severalweek period. ${ }^{20}$ The precise radiobiological mechanism that mediates tumor control in VS remains unknown. ${ }^{25}$ Many of the theories to explain the efficacy of radiation in VS have been extrapolated from the study of malignant tumors, which is problematic given contrasting biological behavior. Because cells are most sensitive to ionizing radiation during mitosis, one could hypothesize that tumors with a large population of proliferating cells would be more susceptible to radiation-induced apoptosis compared with slow-growing tumors. Alternatively, tumors with rapid growth may exceed the capacity of the local vascular distribution, resulting in tumor hypoxia and radioresistance, suggesting that slow-growing tumors would be more susceptible than fast-growing VS. Specific to VS, several studies have demonstrated that low-dose radiation has little direct effect on VS cells but secondarily impedes tumor growth through endothelial injury and arterial thrombosis. . $^{13,14}$

Given the controversies surrounding the true efficacy of SRS for growing VSs, we sought to review our experience over the last decade, during which we used contemporary low-dose treatment parameters. Herein, we present the results of treatment in 68 patients who ultimately received Gamma Knife radiosurgery (GKS) for sporadic VS after an initial wait-and-scan approach failed in tumors that demonstrated growth of at least $1 \mathrm{~mm} /$ year.

\section{Methods \\ Data Collection}

After institutional review board approval, a prospective VS clinical database was queried, and all patients who received SRS for sporadic VS between 2004 and 2014 at a single large tertiary academic referral center were initially evaluated. All tumors that demonstrated $>1 \mathrm{~mm} /$ year linear growth and that subsequently underwent SRS following an initial observation period of at least 6 months were included in the study. Patients with a history of neurofibromatosis Type 2, macrocystic features, prior treatment, or $<14$ months of post-SRS radiographic follow-up were excluded.

Data pertaining to tumor laterality, location, size, growth, Gamma Knife dose parameters, need for salvage treatment, and hearing status were collected. Radiosurgical data points included tumor volume treated, dose to the tumor margin, maximum tumor dose, and number of isocenters. Tumor size, location, and hearing outcomes were reported according to the 1995 American Academy of Otolaryngology-Head and Neck Surgery (AAO-HNS) guidelines. ${ }^{5}$ Patients with serviceable hearing (Class A or B) at the time of SRS were included in audiometric analysis. Tumor measurements were made on axial T1-weighted
post-Gd MRI scans by an otolaryngologist (A.P.M.) and neurosurgeon (M.J.L.). For VSs that were exclusively intracanalicular (IC), the size of the tumor was measured along the length of the internal auditory canal. For tumors with extracanalicular (EC) extension, the portion within the cerebellopontine angle was measured in 2 axial planes: one parallel to the posterior axis of the petrous bone and the other perpendicular to the posterior axis of the petrous bone. The EC tumor size was then calculated by taking the square root of the product of these 2 diameters. ${ }^{5}$

\section{Treatment and Surveillance}

At our institution, patients are considered for wait-andscan management in the setting of sporadic, minimally symptomatic IC VSs and EC tumors $<2 \mathrm{~cm}$ in maximum posterior fossa diameter. Additional factors that influence initial management include patient preference, age, comorbid medical conditions, presence of symptoms attributable to mass effect, and hearing status of the contralateral ear without tumor. For patients who elect an initial wait-and-scan approach but who ultimately demonstrate tumor growth on follow-up imaging, it is recommended that they undergo treatment with microsurgery or SRS. Microsurgery is commonly recommended for younger patients, those with peritumoral or intratumoral macrocysts, and those with symptoms associated with mass effect, whereas radiosurgery is often advised for growing tumors in older patients, those with advanced medical comorbidities, or those with minimal symptomatology.

We routinely obtain imaging at 6 months after diagnosis of VS for patients who elect initial observation. Tumors that demonstrate stable size at the first 6-month scan are then followed annually with MRI. Posttreatment tumor growth or shrinkage was determined by a $\geq 2-\mathrm{mm}$ increase or decrease, respectively.

Stereotactic radiosurgery was performed with either the model 4C or Perfexion (after 2007) Gamma Unit (Elekta $\mathrm{AB}$ ), as previously described..$^{10}$ Following placement of the Leksell model G stereotactic head frame (Elekta AB) under local anesthesia, 1-mm-thick axial spoiled gradient-recalled acquisition high-resolution post-Gd MRI through the posterior fossa is obtained on a 1.5-T scanner. Additionally, a CT scan of the temporal bones (also 1 -mm-thick axial slices) is performed to better visualize the inner ear structures and to optimize stereotactic accuracy. Multiple-isocenter, conformal dose planning was performed using the Leksell GammaPlan software (Elekta $A B)$ with the aid of the imaging set, including coronal and sagittal reconstructions.

Patients were routinely seen every 6 months for the 1st year after treatment, then annually for the next 3 years, and at least biennially thereafter. Follow-up intervals were shortened in cases of new symptoms or radiographic evidence of progression. Tumor surveillance imaging was performed at each follow-up interval, using thin-slice contrast-enhanced T1-weighted MRI.

\section{Statistical Analysis}

Continuous features were summarized with medians, interquartile ranges (IQRs), and ranges; categorical 
features were summarized with frequency counts and percentages. Comparisons of pretreatment growth rates, brainstem contact, fundal cap, and tumor size at treatment among tumor outcomes (decrease vs stable, vs increase or combined decrease and stable, vs increase) and between patient groups of interest were evaluated using KruskalWallis, Wilcoxon rank-sum, and Fisher exact tests. The association of pretreatment growth rates with tumor increase was also evaluated using a logistic regression model, and summarized with an odds ratio and 95\% confidence interval. Survival free of revision treatment was estimated using the Kaplan-Meier method. The duration of followup for survival free of revision was calculated from the treatment date to revision date or the last MRI study date. Statistical analyses were performed using version 9.3 of the SAS software package (SAS Institute). All tests were 2 -sided, and $\mathrm{p}$ values $<0.05$ were considered statistically significant.

\section{Results \\ Study Population}

Between 2004 and 2014, a total of 432 patients with VSs were treated with SRS at our institution. During this time period, 68 patients with unilateral VS met study criteria. Specifically, these patients had documented tumor growth rates exceeding $1 \mathrm{~mm} /$ year during the observation period and were subsequently treated with SRS (Table 1). The median patient age at the time of SRS was 67.0 years (range 23-88 years). During the observation and follow-up periods a total of 473 MRI studies were reviewed. Forty patients (59\%) were female and $28(41 \%)$ were male. The median pretreatment observation period was 16 months (range 6-80 months), and the median posttreatment follow-up period was 43.5 months (range 14-147 months). The median tumor volume treated was $807 \mathrm{~mm}^{3}$ (range $\left.97-11,000 \mathrm{~mm}^{3}\right)$. The median dose to the tumor margin was 13 Gy (range 12-14 Gy), and the median maximum dose was 26 Gy (range 24-28 Gy). The median number of isocenters used was 6 (range 1-15 isocenters).

\section{Tumor Control for EC and IC VSs}

Of the 68 total patients included in the study, 59 (86.8\%) had an EC component at the time of treatment, whereas 9 (13.2\%) had purely IC tumors. Fifty-nine (86.8\%) of the 68 patients with EC and IC lesions achieved tumor control, meaning that tumor growth $>2 \mathrm{~mm}$ was not detected at the last follow-up imaging session, which occurred within a median follow-up period of 43.5 months (range 14-147 months) after primary SRS treatment.

\section{Tumor Control for EC VSs}

Of the 59 tumors that were EC, 15 (25.4\%) began the observation period as IC tumors and later converted to EC tumors prior to SRS. Among all EC tumors, 29 (49.2\%) decreased in size, 21 (35.6\%) were stable, and 9 (15.3\%) increased in size following SRS at the last imaging follow-up, for a tumor control rate of $84.7 \%$ for growing EC tumors (Figs. 1 and 2). The median follow-up duration among the patients with EC tumors was 43 months (range 14-147 months). The combined pretreatment growth rate
TABLE 1. Pretreatment patient characteristics and SRS treatment parameters in 68 patients with VS

\begin{tabular}{lcc}
\hline \multicolumn{1}{c}{ Characteristic } & Value & $\%$ \\
\hline Age in yrs & & \\
\hline Mean (SD) & $65.6(12.0)$ & \\
\hline Median (range) & $67(23-88)$ & \\
\hline Sex & & \\
\hline Male & 28 & 41 \\
\hline Female & 40 & 59 \\
\hline Tumor laterality & & \\
\hline Lt side & 36 & 53 \\
\hline Rt side & 32 & 47 \\
\hline Tumor location & & \\
\hline IC & 9 & \\
\hline EC & 59 & \\
\hline AAO-HNS hearing classification pre-SRS & & \\
\hline A & 14 & \\
\hline B & 18 & 21 \\
\hline C & 3 & 24 \\
\hline D & 17 & \\
\hline No data & 16 & \\
\hline SRS treatment parameters in Gy & $13(12-14)$ & \\
\hline Median dose to margin (range) & $26(22-28)$ & \\
\hline Median maximum dose (range) & & \\
\hline
\end{tabular}

for EC lesions that achieved tumor control was $2.08 \mathrm{~mm} /$ year (IQR 1.59-3.17 mm/year; range 1.10-10.64 mm/ year), whereas the pretreatment growth rate for EC tumors that continued to increase in size following SRS was 3.26 $\mathrm{mm} /$ year (IQR 2.76-5.27 mm/year; range 2.34-5.8 mm/ year) $(\mathrm{p}=0.009)($ Table 2$)$. Based on this result, a pretreatment growth rate of $2.5 \mathrm{~mm} /$ year was used to best separate the observational cohort based on probability of posttreatment tumor growth. As such, control was achieved in 32 of $33(97.0 \%)$ EC tumors with a pretreatment growth rate $<2.5 \mathrm{~mm} /$ year, compared with 18 of $26(69.2 \%) \mathrm{EC}$ lesions with a pretreatment growth rate $\geq 2.5 \mathrm{~mm} /$ year in which tumor control was achieved $(p=0.007)$.

No other feature aside from pretreatment growth rate was found to be significantly associated with tumor control following SRS. Specifically, 19 of 50 (38.0\%) EC tumors that decreased or were stable had brainstem contact at the time of SRS treatment, compared with 2 of $9(22.2 \%)$ tumors that increased in size $(\mathrm{p}=0.47)$. Twenty-eight of $50(56.0 \%)$ EC tumors that decreased or were stable had a fundal cap, compared with 4 of $9(44.4 \%)$ tumors that demonstrated growth $(p=0.72)$. Finally, pretreatment lesion size was not different between EC tumors that were stable or decreased in size versus those that grew. The median EC tumor size for lesions that were controlled was $8.58 \mathrm{~mm}$ (IQR 5.54-11.57 mm; range 2.97-24.88 mm) versus $7.98 \mathrm{~mm}$ (IQR 7.21-10.07 mm; range 5.72-12.67 $\mathrm{mm})(\mathrm{p}=0.91)$ for tumors that failed treatment.

Nine EC tumors increased in size by $>2 \mathrm{~mm}$ and demonstrated ongoing sequential growth at the most recent follow-up imaging study. Of these 9 patients, 5 had 4 MR 


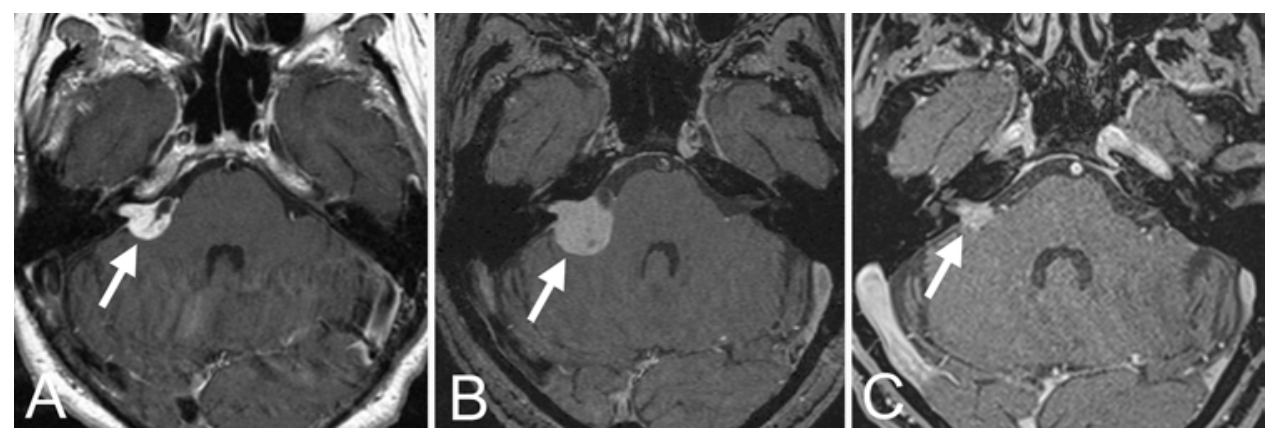

FIG. 1. Serial axial contrast-enhanced MRI studies demonstrating significant tumor shrinkage following GKS for a slow-growing (< $3 \mathrm{~mm} /$ year) right-sided VS with cisternal extension (white arrows). A: Initial imaging revealed a 13.1-mm right-sided VS that grew $5.9 \mathrm{~mm}$ over the course of 27 months. B: Planning MRI demonstrated progressive growth with increasing mass effect. C: Following SRS using a 13-Gy marginal dose prescribed to the 50\% isodose line, the tumor decreased in size over a period of 63 months of follow-up.

scans and 4 had 3 MR scans following SRS at a median follow-up period of 38 months (range 24.5-116 months). Importantly, all patients who were found to have posttreatment growth had a minimum follow-up of 2 years, minimizing the risk for mistaking tumor growth for transient post-SRS tumor swelling. ${ }^{18}$ Five of $9(55.6 \%)$ patients required a secondary treatment at 2.2, 2.7,3.1,3.8, and 6.6 years following SRS treatment. Three patients had repeat SRS and 2 had salvage microsurgery at a median interval of 3.1 years (range 2.2-6.6 years) following the initial radiosurgical treatment. Among the EC tumor group, the estimated rates of survival free of revision SRS or salvage surgery (expressed as 95\% CI; number still at risk) at 1, 3, 5 , and 7 years following the primary SRS treatment were $100 \%$ (100-100; 59), 96\% (90-100; 39), 90\% (80-100; 19), and $80 \%(62-100 ; 6)$, respectively, with a median followup duration of 3.6 years for the 54 tumors that did not require revision intervention (IQR 2.2-5.2 years; range 1.112.1 years) (Fig. 3). Of the 4 patients with EC tumors that grew $>2 \mathrm{~mm}$ but who did not undergo revision treatment, all 4 were without severe symptoms or associated neurological sequelae and chose follow-up with repeat imaging.

\section{Tumor Control for IC VSs}

Fifteen $(62.5 \%)$ of the 24 tumors that were originally IC became EC tumors at a median of 1.6 years (IQR 1.02.2 years; range $0.5-6.1$ years) after their initial diagno- sis during the observation period. Of the 9 IC tumors that were treated with SRS, 2 (22.2\%) ultimately decreased in size and $7(77.8 \%)$ were stable at a median follow-up period of 48.5 months (range 24-97 months) (Fig. 4). In all lesions that were IC at the time of SRS, tumor control was achieved during the follow-up period.

\section{Postradiosurgery Tumor Swelling}

Patients were considered to have tumor swelling secondary to treatment effect if there was $\geq 2 \mathrm{~mm}$ of tumor enlargement measured at the first or second follow-up MR scan completed at approximately 6 months and 12 months, respectively, after the date of radiosurgery, followed by a decrease or stabilization in tumor size on subsequent follow-up imaging. ${ }^{18}$ Six of $59(10.2 \%)$ patients treated for EC tumors had postradiosurgery tumor swelling at a median of 6 months (range 5-12 months) following SRS treatment. The mean maximum tumor swelling was $2.8 \pm 1.1$ $\mathrm{mm}$ (mean $\pm \mathrm{SD})$. All $6(100 \%)$ patients with EC lesions experienced resolution of the transient tumor swelling at a median of 25 months (range 11-61 months) following SRS treatment.

\section{Cranial Nerve and Hearing Outcomes}

No patient had facial nerve weakness, trigeminal nerve symptoms, or other localizing neurological findings prior
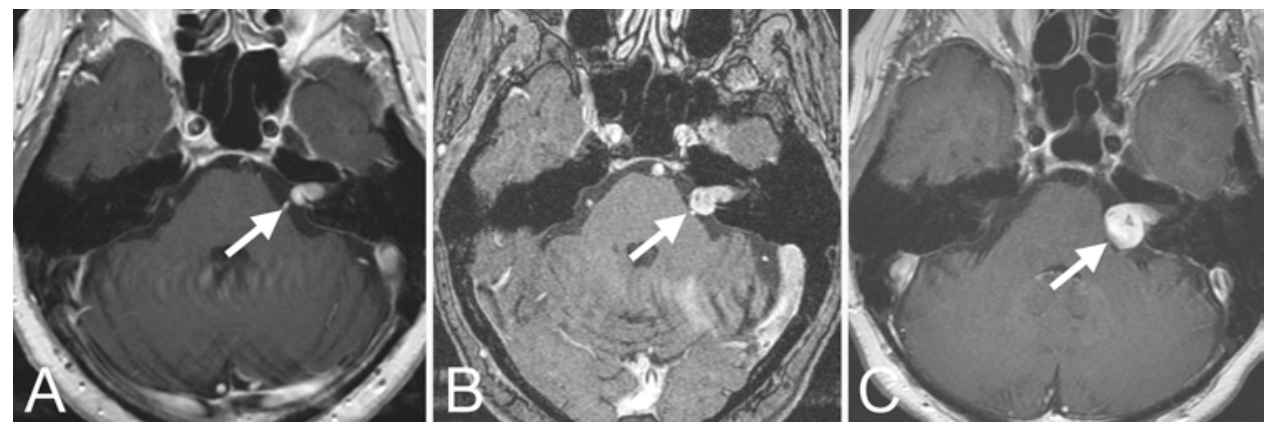

FIG. 2. Serial axial contrast-enhanced MRI studies demonstrating progressive tumor growth following GKS for a fast-growing ( $\geq$ $3 \mathrm{~mm} /$ year) left-sided VS with cisternal extension (white arrows). A: Initial imaging revealed a 3.1-mm left-sided VS that grew 3.4 $\mathrm{mm}$ over the course of 9 months. B: Planning MRI demonstrated progressive growth into the cerebellopontine angle. C: Following SRS using a marginal dose of 12 Gy prescribed to the $50 \%$ isodose line, the tumor increased $4.1 \mathrm{~mm}$ in size over a period of 35 months of follow-up. 
TABLE 2. Comparison of pretreatment growth rates among tumor outcomes for groups of interest

\begin{tabular}{lrll}
\hline \multicolumn{1}{c}{ Group } & $\begin{array}{c}\text { No. of } \\
\text { Pts }\end{array}$ & $\begin{array}{c}\text { Median Pretreatment Growth } \\
\text { Rate in mm/yr (IQR; range) }\end{array}$ & $\begin{array}{c}\mathrm{p} \\
\text { Value }\end{array}$ \\
\hline Treated as EC & 59 & & \\
\hline Decrease & 29 & $2.07(1.59-2.45 ; 1.12-9.86)$ & 0.024 \\
\hline Stable & 21 & $2.75(1.50-3.46 ; 1.10-10.64)$ & \\
\hline Increase & 9 & $3.26(2.76-5.27 ; 2.34-5.80)$ & \\
\hline Treated as EC & 59 & & \\
\hline Decrease \& stable & 50 & $2.08(1.59-3.17 ; 1.10-10.64)$ & 0.009 \\
\hline Increase & 9 & $3.26(2.76-5.27 ; 2.34-5.80)$ & \\
\hline IC & 9 & & $\mathrm{NE}$ \\
\hline Decrease & 2 & $1.13,3.15^{*}$ & \\
\hline Stable & 7 & $1.85(1.59-3.93 ; 1.21-5.04)$ & \\
\hline IC $\rightarrow$ EC $\dagger$ & 15 & & $\mathrm{NE}$ \\
\hline Decrease & 6 & $2.03(1.69-4.02 ; 1.59-5.29)$ & \\
\hline Stable & 7 & $2.75(1.74-6.34 ; 1.30-10.64)$ & \\
\hline Increase & 2 & $2.34,5.27^{*}$ & \\
\hline
\end{tabular}

$\mathrm{NE}=$ not evaluated; $\mathrm{Pts}=$ patients

* The growth values (in $\mathrm{mm} / \mathrm{yr}$ ) are shown for the 2 patients in the group.

No other statistical analysis was performed in these groups due to the small sample size.

† Arrow signifies conversion from IC to EC.

to or following SRS. Of the 9 patients with tumor growth following SRS, 4 had subjective balance difficulty prior to SRS and 3 had ongoing balance difficulty post-SRS while their tumors continued to grow. There was no clear difference in associated symptoms between tumors growing < $2.5 \mathrm{~mm} /$ year and those growing $\geq 2.5 \mathrm{~mm} /$ year. A total of 33 patients had serviceable hearing (AAO-HNS Class A or B) at the time of GKS. Twenty-two patients did not have post-SRS audiogram data available. Of the 11 patients with both pre- and post-SRS audiogram data, all exhibited tumor control, with $4(36.4 \%)$ who maintained serviceable hearing and 7 (63.6\%) who developed nonserviceable hearing at a median follow-up period of 24 months (range 9-92 months).

\section{Discussion}

The current study was designed to address the impact of pretreatment growth rate on post-SRS tumor control in a large cohort of patients who initially selected a wait-andscan treatment approach. These data demonstrate that for EC tumors, pretreatment growth rate is a strong predictor of tumor control. Specifically, EC VSs that stabilized or decreased in size following SRS grew at a median rate of $2.08 \mathrm{~mm} /$ year before treatment, whereas the tumors that increased in size following radiation had a median pretreatment growth rate of $3.26 \mathrm{~mm} /$ year $(\mathrm{p}=0.009)$. Furthermore, when the preirradiation growth rate of an observed EC VS was $\geq 2.5 \mathrm{~mm} /$ year versus $<2.5 \mathrm{~mm} /$ year, GKS was found to fail in $31 \%$ versus $3 \%$ of patients, respectively $(p=0.007)$.

Due to heterogeneous study populations and limited patient numbers, previous studies have published conflicting data concerning the influence of preirradiation tumor

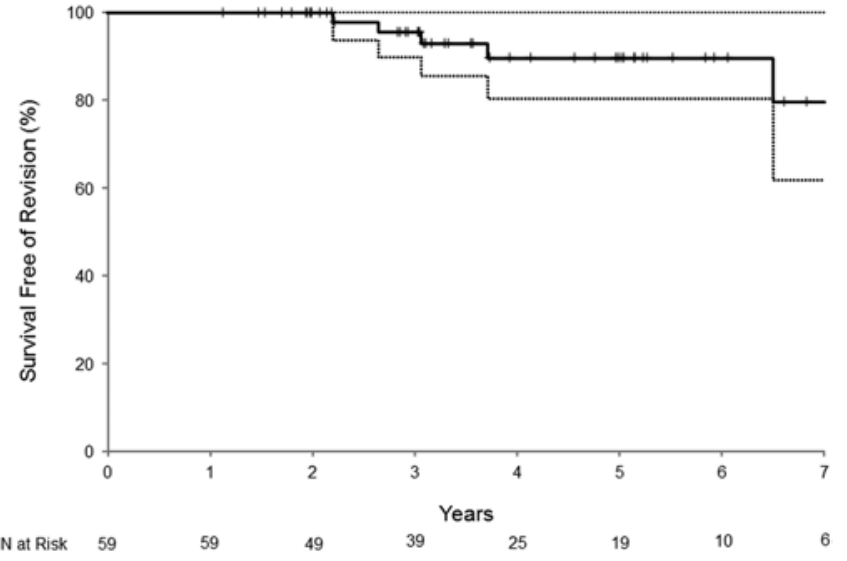

FIG. 3. Kaplan-Meier plot demonstrating retreatment-free survival following SRS of 59 growing sporadic VSs that demonstrated EC extension at time of diagnosis (solid line denotes survival curve; dotted line shows the $95 \% \mathrm{Cl}$; vertical tick-marks indicate censored data).

growth on treatment response. In 2011, Timmer et al. compared SRS outcomes between 67 patients with proven tumor growth and 33 patients in whom the history of growth was unknown. ${ }^{26}$ After a mean follow-up of 26 months there was no difference between groups with respect to rate of SRS failure. In 2012, Varughese and colleagues analyzed GKS treatment outcomes in 45 patients with growing VS. ${ }^{28}$ At a mean follow-up of 50 months, they found that a slower pretreatment growth rate was associated with better rates of tumor control; however, this relationship did not achieve statistical significance $(\mathrm{p}=0.06)$. In 2014, Niu et al. investigated 58 patients without neurofibromatosis Type 2, 17 of whom had a prior resection, who were treated with either proton or photon therapy in which a single fraction or fractionated scheme was used. ${ }^{19}$ At a median follow-up of 27 months, they found that stable or shrinking posttreatment status was significantly associated with slower preradiation growth rates. The VSs that had postirradiation expansion $>120 \%$ of their volumetric size at the time of treatment versus nonexpanding tumors were found to have preirradiation volumetric growth rates of $89 \% /$ year and $41 \% /$ year, respectively.

Building on these prior publications, we found a statistically significant association between pretreatment growth and postradiation tumor progression in a large population of untreated sporadic EC VS receiving contemporary single-fraction GKS. In patients with EC VS and tumor growth $\geq 2.5 \mathrm{~mm} /$ year during the observation period, treatment with microsurgery rather than SRS is given consideration on a case-by-case basis. During pretreatment patient counseling in cases in which more rapid growth has been detected, we are now careful to review the results of this study to improve informed decision making. In our practice, we do not currently advocate for higher doses of radiation in faster-growing tumors; however, the goal of future research will be to determine the optimal treatment modality for patients based on tumor growth characteristics.

In addition to the predictive value of pretreatment growth rate on tumor control after SRS, we sought to investigate the impact of tumor size, brainstem contact, 


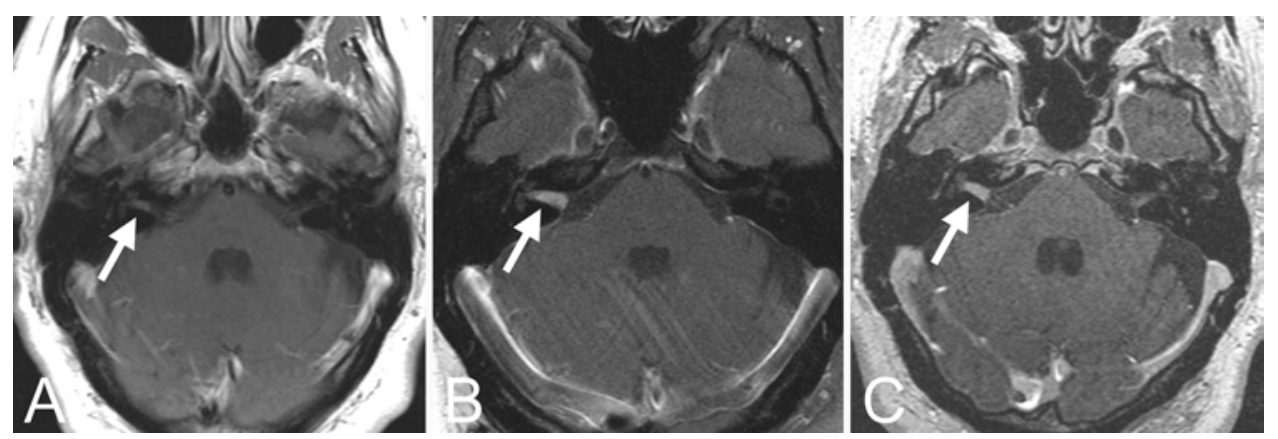

FIG. 4. Serial axial contrast-enhanced MRI studies demonstrating successful tumor control following GKS for a fast-growing ( $\geq$ $3 \mathrm{~mm} /$ year) right-sided IC VS (white arrows). A: Initial imaging revealed a 5.7- $\mathrm{mm}$ right-sided IC VS that grew $4.3 \mathrm{~mm}$ over the course of 10 months. B: Planning MRI demonstrated progressive growth extending to the porus acusticus. C: Following SRS using a marginal dose of 12.5 Gy prescribed to the $50 \%$ isodose line, the tumor has remained radiologically stable over a period of 26 months of follow-up.

and the presence of a fundal cap at the time of radiation therapy. To date, conflicting reports exist with regard to the predictive value of these factors and post-SRS tumor control. In a study by Hasegawa et al., a VS volume of $>15$ $\mathrm{cm}^{3}$ was significantly associated with radiologically confirmed growth following treatment, whereas Varughese et al. found that patients with larger tumors had the highest odds of tumor control., ${ }^{9,28}$ In a prior analysis we also found that larger tumors had decreased tumor control. ${ }^{17}$ However, in the present study, which only included tumors with documented pretreatment growth, neither brainstem contact, pretreatment tumor size, nor the presence of a fundal cap were found to be predictive of tumor control following radiosurgery.

When analyzing tumor control following SRS, the phenomenon of transient posttreatment tumor expansion must be addressed. Currently, there is no consensus regarding how to define tumor expansion due to treatment effect versus growth due to true tumor progression. The reported frequency of transient tumor swelling following SRS ranges from 3\% to 74\%, with most tumors reaching a maximum volume after a median of 5 months and the first signs of regression occurring at a median of 15 months. ${ }^{26,27}$ In the present study, $6(10.2 \%)$ of 59 patients with an EC tumor experienced enlargement by $\geq 2 \mathrm{~mm}$ at the time of the initial 6-month post-SRS MRI session. Of these 6 patients, all lesions demonstrated regression and were later categorized as tumors that were stable or decreased in size. Transient tumor swelling resolved at a median of 25 months following SRS. None of the 9 EC tumors in which SRS failed in the present study grew by $\geq 2 \mathrm{~mm}$ at the time of the 6-month posttreatment scan. As such, early posttreatment tumor swelling does not correlate with eventual radiosurgical failure in our experience, and extreme caution must be exercised when increased volume of the treated tumor is seen within the first 12 months, because this rarely represents true treatment failure. Furthermore, it is apparent that the 9 patients in the current study in whom SRS failed represent cases of real tumor growth, with a median follow-up duration of 38 months, minimum follow-up of 24 months, and $\geq 2$ posttreatment MR scans. This follow-up duration falls well outside of the time course for posttreatment tumor swelling as described by previous studies.
Although variable VS growth rates are reported in the literature, several large natural history studies have demonstrated convincing evidence that a significant portion of VSs do not grow for extended periods of time following initial diagnosis. ${ }^{2,21,24}$ This knowledge has led many centers to adopt an initial wait-and-scan protocol for small to medium-sized sporadic VS. Stangerup et al. published data on 552 patients with VS in whom observation was elected and found that only $17 \%$ of intrameatal and $28.9 \%$ of extrameatal tumors displayed growth during a follow-up of 3.6 years. ${ }^{24}$ In 2005 Smouha et al. reported comparable data based on a meta-analysis comprising 21 studies and 1345 patients, in which they showed that $57 \%$ of newly diagnosed tumors did not grow or regressed during the first 3 years following VS diagnosis. ${ }^{23}$ These data have led many to conclude that published rates of SRS tumor control are inflated, because a significant portion of tumors in most series were never destined to grow during the typical follow-up period. ${ }^{16}$ When analyzing a population of patients with IC and EC VS that demonstrated clear radiological growth prior to SRS, we found that 59 of $68(87 \%)$ of tumors demonstrated radiological control at a median follow-up of 43.5 months, which is remarkably similar to the estimate of $85.4 \%$ at 4 years by Varughese and colleagues. ${ }^{28}$ Thus, patients with growing tumors should be counseled differently regarding the success of SRS, compared with those in whom growth status is unknown.

The issue of preoperative growth as a potential predictor of radiosurgical failure leads the multidisciplinary VS team to consider the following questions. When is the optimal timing of treatment? Does waiting for documented growth or symptom progression increase the risk of future treatment failure? Several groups advise that up-front "proactive" SRS offers the greatest chance for tumor control and function preservation, whereas others contend that this strategy stacks the deck in favor of SRS by including a large number of tumors that were not destined to grow. ${ }^{22}$ Alternatively, given the inherent risk of SRS failure in fast-growing tumors, one might consider microsurgery for tumors growing $\geq 2.5 \mathrm{~mm} / \mathrm{year}$. It has been our experience that most patients who start with a conservative wait-and-scan approach ultimately pursue SRS when there is evidence of growth on serial imaging. Ultimately, each 
patient must be assessed individually, and a decision regarding management should be made after assessing the growth rate of the tumor, patient preference, and attendant risks of treatment.

Among all patients with IC and EC tumors in the present study who had AAO-HNS Class A or B hearing with available pre- and post-SRS audiograms, the hearing preservation rate at a median of 24 months was $36 \%$. Frustratingly, audiometric follow-up was not available for approximately $50 \%$ of patients who started with serviceable hearing in this study. In a meta-analysis published in 2010, Yang et al..$^{29}$ reported a 51\% hearing preservation rate at a median follow-up just under 3 years, and in 2013 Carlson et al. reported rates of preserved serviceable hearing of $55 \%$ at 3 years. ${ }^{3}$ The higher percentage of patients who lost serviceable hearing following radiation in the current study is most likely attributable to the added effects of tumor growth. ${ }^{1}$ Further investigation and better audiometric follow-up will be necessary in the future to better understand the impact of tumor growth on hearing outcomes.

In closing, several strengths and limitations of the current study deserve mention. To our knowledge, this is the largest study to date to specifically assess the efficacy of SRS in a cohort of patients with growing sporadic VS. We only included previously untreated tumors that were then treated with contemporary dosimetry and planning software. Limitations of the present study include a treatment selection bias, short duration of observed tumor growth in several patients prior to SRS, several patients with postSRS follow-up of $<2$ years, and lack of consistent audiometry data. In patients with observation of $<1$ year, one may argue that it was truly too early to declare failed conservative management. ${ }^{6,21}$ In the current study, only those patients who demonstrated a minimum pretreatment growth rate of $1 \mathrm{~mm} /$ year underwent treatment, which we believe accurately reflects the threshold for most centers to recommend an intervention. The lack of volumetric measurements imparts a certain degree of error because VSs are $3 \mathrm{D}$ structures. However, the precedent to use a linear size difference of $\geq 2 \mathrm{~mm}$ to distinguish growth and shrinkage is well established in the literature. ${ }^{1,5,9,11}$ Furthermore, in an analysis by Fiirgaard et al. comparing VS tumor volumes to the maximal tumor diameter, the authors found good concordance between 2D and 3D measurements and concluded that the linear diameter is an accurate and more practical parameter for tumor size comparison. ${ }^{7}$

\section{Conclusions}

Overall, SRS is effective in treating VSs in which initial observation fails. The rate of growth and location of a growing tumor prior to radiosurgery are strong predictors of tumor control. Stereotactic radiosurgery is successful at halting tumor progression in $97 \%$ of growing EC tumors that exhibit pretreatment growth $<2.5 \mathrm{~mm} /$ year. However, it is only $69 \%$ successful in treating EC tumors exhibiting initial rapid growth ( $\geq 2.5 \mathrm{~mm} /$ year). No growing IC VS displayed treatment failure following SRS in this patient cohort. These data may help guide patient counseling regarding treatment choice following failed observation.

\section{Acknowledgments}

Internal departmental funding was used without commercial sponsorship or support.

\section{References}

1. Aoyama H, Onodera S, Takeichi N, Onimaru R, Terasaka S, Sawamura Y, et al: Symptomatic outcomes in relation to tumor expansion after fractionated stereotactic radiation therapy for vestibular schwannomas: single-institutional long-term experience. Int J Radiat Oncol Biol Phys 85:329-334, 2013

2. Bakkouri WE, Kania RE, Guichard JP, Lot G, Herman P, Huy PT: Conservative management of 386 cases of unilateral vestibular schwannoma: tumor growth and consequences for treatment. J Neurosurg 110:662-669, 2009

3. Carlson ML, Jacob JT, Pollock BE, Neff BA, Tombers NM, Driscoll CL, et al: Long-term hearing outcomes following stereotactic radiosurgery for vestibular schwannoma: patterns of hearing loss and variables influencing audiometric decline. J Neurosurg 118:579-587, 2013

4. Carlson ML, Tveiten OV, Driscoll CL, Goplen FK, Neff BA, Pollock BE, et al: Long-term quality of life in patients with vestibular schwannoma: an international multicenter crosssectional study comparing microsurgery, stereotactic radiosurgery, observation, and nontumor controls. J Neurosurg 122:833-842, 2015

5. Committee on Hearing and Equilibrium: Committee on Hearing and Equilibrium guidelines for the evaluation of hearing preservation in acoustic neuroma (vestibular schwannoma). Otolaryngol Head Neck Surg 113:179-180, 1995

6. Deen HG, Ebersold MJ, Harner SG, Beatty CW, Marion MS, Wharen RE, et al: Conservative management of acoustic neuroma: an outcome study. Neurosurgery 39:260-266, 1996

7. Fiirgaard B, Pedersen CB, Lundorf E: The size of acoustic neuromas: CT and MRI. Neuroradiology 39:599-601, 1997

8. Gauden A, Weir P, Hawthorne G, Kaye A: Systematic review of quality of life in the management of vestibular schwannoma. J Clin Neurosci 18:1573-1584, 2011

9. Hasegawa T, Fujitani S, Katsumata S, Kida Y, Yoshimoto M, Koike J: Stereotactic radiosurgery for vestibular schwannomas: analysis of 317 patients followed more than 5 years. Neurosurgery 57:257-265, 2005

10. Jacob JT, Carlson ML, Schiefer TK, Pollock BE, Driscoll CL, Link MJ: Significance of cochlear dose in the radiosurgical treatment of vestibular schwannoma: controversies and unanswered questions. Neurosurgery 74:466-474, 2014

11. Kanzaki J, Tos M, Sanna M, Moffat DA, Monsell EM, Berliner KI: New and modified reporting systems from the consensus meeting on systems for reporting results in vestibular schwannoma. Otol Neurotol 24:642-649, 2003

12. Lau T, Olivera R, Miller T Jr, Downes K, Danner C, van Loveren HR, et al: Paradoxical trends in the management of vestibular schwannoma in the United States. J Neurosurg 117:514-519, 2012

13. Lee DJ, Westra WH, Staecker H, Long D, Niparko JK, Slattery WH III: Clinical and histopathologic features of recurrent vestibular schwannoma (acoustic neuroma) after stereotactic radiosurgery. Otol Neurotol 24:650-660, 2003

14. Linskey ME, Martinez AJ, Kondziolka D, Flickinger JC, Maitz AH, Whiteside T, et al: The radiobiology of human acoustic schwannoma xenografts after stereotactic radiosurgery evaluated in the subrenal capsule of athymic mice. J Neurosurg 78:645-653, 1993

15. Meyer TA, Canty PA, Wilkinson EP, Hansen MR, Rubinstein JT, Gantz BJ: Small acoustic neuromas: surgical outcomes versus observation or radiation. Otol Neurotol 27:380-392, 2006

16. Miller T, Lau T, Vasan R, Danner C, Youssef AS, van Loveren $\mathrm{H}$, et al: Reporting success rates in the treatment of 
vestibular schwannomas: are we accounting for the natural history? J Clin Neurosci 21:914-918, 2014

17. Milligan BD, Pollock BE, Foote RL, Link MJ: Long-term tumor control and cranial nerve outcomes following Gamma Knife surgery for larger-volume vestibular schwannomas. J Neurosurg 116:598-604, 2012

18. Mindermann T, Schlegel I: How to distinguish tumor growth from transient expansion of vestibular schwannomas following Gamma Knife radiosurgery. Acta Neurochir (Wien) 156:1121-1123, 2014

19. Niu NN, Niemierko A, Larvie M, Curtin H, Loeffler JS, McKenna MJ, et al: Pretreatment growth rate predicts radiation response in vestibular schwannomas. Int J Radiat Oncol Biol Phys 89:113-119, 2014

20. Pollock BE, Lunsford LD: A call to define stereotactic radiosurgery. Neurosurgery 55:1371-1373, 2004

21. Raut VV, Walsh RM, Bath AP, Bance ML, Guha A, Tator $\mathrm{CH}$, et al: Conservative management of vestibular schwannomas-second review of a prospective longitudinal study. Clin Otolaryngol Allied Sci 29:505-514, 2004

22. Régis J, Carron R, Park MC, Soumare O, Delsanti C, Thomassin JM, et al: Wait-and-see strategy compared with proactive Gamma Knife surgery in patients with intracanalicular vestibular schwannomas: clinical article. J Neurosurg 119 Suppl:105-111, 2013

23. Smouha EE, Yoo M, Mohr K, Davis RP: Conservative management of acoustic neuroma: a meta-analysis and proposed treatment algorithm. Laryngoscope 115:450-454, 2005

24. Stangerup SE, Caye-Thomasen P, Tos M, Thomsen J: The natural history of vestibular schwannoma. Otol Neurotol 27:547-552, 2006

25. Sweeney P, Yajnik S, Hartsell W, Bovis G, Venkatesan J: Stereotactic radiotherapy for vestibular schwannoma. Otolaryngol Clin North Am 42:655-663, 2009

26. Timmer FC, Mulder JJ, Hanssens PE, van Overbeeke JJ, Donders RT, Cremers CW, et al: Gamma Knife radiosurgery for vestibular schwannomas: identification of predictors for continued tumor growth and the influence of documented tumor growth preceding radiation treatment. Laryngoscope 121:1834-1838, 2011

27. van de Langenberg R, Dohmen AJ, de Bondt BJ, Nelemans PJ, Baumert BG, Stokroos RJ: Volume changes after stereotactic LINAC radiotherapy in vestibular schwannoma: control rate and growth patterns. Int J Radiat Oncol Biol Phys 84:343-349, 2012

28. Varughese JK, Wentzel-Larsen T, Pedersen PH, Mahesparan R, Lund-Johansen M: Gamma Knife treatment of growing vestibular schwannoma in Norway: a prospective study. Int $\mathbf{J}$ Radiat Oncol Biol Phys 84:e161-e166, 2012

29. Yang I, Sughrue ME, Han SJ, Aranda D, Pitts LH, Cheung SW, et al: A comprehensive analysis of hearing preservation after radiosurgery for vestibular schwannoma. J Neurosurg 112:851-859, 2010

\section{Disclosures}

The authors report no conflict of interest concerning the materials or methods used in this study or the findings specified in this paper.

\section{Author Contributions}

Conception and design: Link, Marston, Jacob. Acquisition of data: Link, Marston, Jacob. Analysis and interpretation of data: Link, Marston, Jacob, Carlson. Drafting the article: Link, Marston, Jacob, Carlson, Pollock. Critically revising the article: all authors. Reviewed submitted version of manuscript: all authors. Approved the final version of the manuscript on behalf of all authors: Link. Statistical analysis: Link, Marston, Jacob, Carlson. Administrative/technical/material support: Link. Study supervision: Link.

\section{Correspondence}

Michael J. Link, Department of Neurologic Surgery, Mayo Clinic, 200 First St. SW, Rochester, MN 55905. email: link.michael@ mayo.edu. 